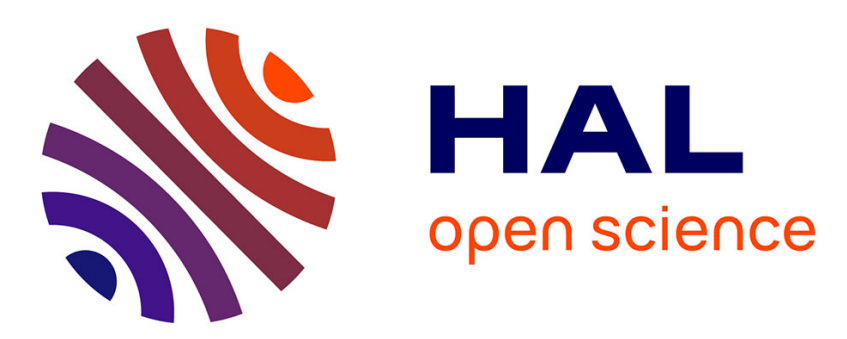

\title{
Single station determination of Rayleigh wave ellipticity by using the random decrement technique (RayDec)
}

\author{
Manuel Hobiger, Pierre-Yves Bard, Cécile Cornou, Nicolas Le Bihan
}

\section{To cite this version:}

Manuel Hobiger, Pierre-Yves Bard, Cécile Cornou, Nicolas Le Bihan. Single station determination of Rayleigh wave ellipticity by using the random decrement technique (RayDec). Geophysical Research Letters, 2009, 36, pp.L14303. 10.1029/2009GL038863 . hal-00413407

\section{HAL Id: hal-00413407 https://hal.science/hal-00413407}

Submitted on 29 Oct 2021

HAL is a multi-disciplinary open access archive for the deposit and dissemination of scientific research documents, whether they are published or not. The documents may come from teaching and research institutions in France or abroad, or from public or private research centers.
L'archive ouverte pluridisciplinaire HAL, est destinée au dépôt et à la diffusion de documents scientifiques de niveau recherche, publiés ou non, émanant des établissements d'enseignement et de recherche français ou étrangers, des laboratoires publics ou privés. 


\title{
Single station determination of Rayleigh wave ellipticity by using the random decrement technique (RayDec)
}

\author{
M. Hobiger, ${ }^{1}$ P.-Y. Bard, ${ }^{1}$ C. Cornou, ${ }^{1}$ and N. Le Bihan ${ }^{2}$ \\ Received 24 April 2009; revised 13 June 2009; accepted 22 June 2009; published 28 July 2009.
}

[1] Rayleigh wave ellipticity as a function of frequency is closely linked to underground structure, i.e., shear wave velocity profile and sediment thickness. The possibility to calculate these underground properties by inverting ellipticity curves has recently been shown. We propose a new technique enabling the Rayleigh wave ellipticity to be recovered over a wide frequency range by using ambient noise recordings. Based on the random decrement technique commonly used to characterize dynamic parameters of buildings, this method eliminates all wave types except Rayleigh waves. We apply the method to noise synthetics simulated for different underground structures and show its applicability to real seismic noise data. Citation: Hobiger, M., P.-Y. Bard, C. Cornou, and N. Le Bihan (2009), Single station determination of Rayleigh wave ellipticity by using the random decrement technique (RayDec), Geophys. Res. Lett., 36, L14303, doi:10.1029/2009GL038863.

\section{Introduction}

[2] In order to properly evaluate local site amplification it is important to have a good knowledge of the local soil properties, i.e., shear-wave velocities and sediment thickness. These soil properties can be obtained by inverting dispersion curves. One way is to use the dispersion curves of surface waves, as derived from active or passive seismic array measurements. However, it usually provides reliable information at frequencies higher than the resonance frequency and the inverted velocity profiles do not carry information on the deeper structures. Additional information around the resonance frequency can be added by using $\mathrm{H} / \mathrm{V}$ spectra. Recent studies have shown the possibility to evaluate the S-wave velocity profile by inversion of $\mathrm{H} / \mathrm{V}$ spectra [Fäh et al., 2003; Arai and Tokimatsu, 2004] or joint inversion of $\mathrm{H} / \mathrm{V}$ spectra and dispersion curves [Arai and Tokimatsu, 2005; Picozzi et al., 2005]. The classical H/V technique [Nakamura, 1989] calculates the ratio between the horizontal and the vertical spectrum of ambient vibrations recorded on a single three-component seismic sensor by $H / V(f)=\frac{\sqrt{|E(f)|^{2}+|N(f)|^{2}}}{\sqrt{2}|Z(f)|}$ where $E(f), N(f)$ and $Z(f)$ are the spectra of the east-west, north-south and vertical displacements of the sensor. Both Rayleigh and Love waves contribute to the $\mathrm{H} / \mathrm{V}$ spectrum [Bonnefoy-Claudet et al., 2008] and the presence of both surface wave

\footnotetext{
${ }^{1}$ Laboratoire de Géophysique Interne et Tectonophysique, IRD, LCPC, CNRS, Université Joseph Fourier, Grenoble, France.

${ }^{2}$ GIPSA-Lab, CNRS, Grenoble, France.
}

Copyright 2009 by the American Geophysical Union. 0094-8276/09/2009GL038863 types has to be accounted for in inversion algorithms. Therefore, the proportion between Rayleigh and Love waves in the noise wave field has to be inferred or assumed prior to the inversion [Scherbaum et al., 2003; Arai and Tokimatsu, 2004; Köhler et al., 2006]. Extraction of Rayleigh wave ellipticity and its subsequent inversion [Boore and Toksöz, 1969; Fäh et al., 2001; Malischewsky and Scherbaum, 2004] would avoid making such assumptions.

[3] We propose a new method (RayDec) to estimate the Rayleigh wave ellipticity spectrum by using the random decrement technique [Asmussen, 1997]. A usual application of the random decrement technique is the measurement of resonance frequencies and damping parameters of buildings [Dunand, 2005; Michel et al., 2008]. We propose to apply the random decrement technique to threecomponent seismometer records of seismic noise. By using the vertical component as a master trigger and stacking a large number of horizontal and vertical signals, Rayleigh waves will be emphasized with respect to Love and body waves. Therefore, the obtained ellipticity curve will be closer to the true curve than the H/V curve. In this paper, we will present the method, apply it to synthetic seismic noise and finally show its applicability to real seismic noise records with one example.

\section{Methodology}

[4] Assume that a three-component sensor record consists of three time-series of seismic noise $(e(t)$ in east-west direction, $n(t)$ in north-south direction and $v(t)$ in vertical direction). Each of these time-series has a time length $T$ and consists of $N$ data points. In order to extract the ellipticity of Rayleigh waves we suggest the following method, based on the random decrement technique [Asmussen, 1997]. The principles of the method are to emphasize Rayleigh waves with respect to other wave types by summing a large number of specially tuned signal windows, calculate the energy on the vertical and horizontal summed signals and henceforward estimate Rayleigh wave ellipticity.

[5] The first step consists of filtering the signals by using a narrow-band Chebyshev filter of order 4 and bandwidth $d f$ centered on frequency $f$. We use the Chebyshev filter because of its sharpness. Filtering was performed on a linear frequency scale. The resulting signals are called $e_{f}(t), n_{f}(t)$ and $v_{f}(t)$.

[6] Then we search for all times $\tau_{i}$ where the signal on the vertical component changes its sign from negative to positive $\left(v_{f}\left(\tau_{i}\right) \leq 0, v_{f}\left(\tau_{i}+d t\right)>0\right)$. Every time this condition is satisfied, we store signals of length $\Delta$ on all three components, leading to buffered signals $v_{f, b, i}(t)$, 
$e_{f, b, i}(t)$ and $n_{f, b, i}(t)$ defined in terms of the original signals as (for $0 \leq t \leq \Delta$ ):

$$
\begin{aligned}
& v_{f, b, i}(t)=v_{f}\left(\tau_{i}+t\right), \\
& e_{f, b, i}(t)=e_{f}\left(\tau_{i}-\frac{1}{4 f}+t\right), \\
& n_{f, b, i}(t)=n_{f}\left(\tau_{i}-\frac{1}{4 f}+t\right) .
\end{aligned}
$$

The shift of $\frac{1}{4 f}$ on the eastern and northern components accounts for the $90^{\circ}$ phase shift between the vertical and horizontal components of Rayleigh waves. Then we build a horizontal signal $h_{f, b, i}(t)$ by projecting the east-west and north-south components onto an axis forming an azimuth angle $\vartheta_{i}$ with the northern direction by

$$
h_{f, b, i}(t)=\sin \left(\vartheta_{i}\right) \cdot e_{f, b, i}(t)+\cos \left(\vartheta_{i}\right) \cdot n_{f, b, i}(t) .
$$

The angle $\vartheta_{i}$ is chosen in such a way that it maximizes the correlation between the vertical signal $v_{f, b, i}(t)$ and the horizontal signal $h_{f, b, i}(t)$ given by:

$$
\begin{aligned}
C_{f, b, i}\left(\vartheta_{i}\right)= & \int_{0}^{\Delta} v_{f, b, i}(t) \cdot h_{f, b, i}(t) d t \\
C_{f, b, i}\left(\vartheta_{i}\right)= & \sin \left(\vartheta_{i}\right) \int_{0}^{\Delta} v_{f, b, i}(t) \cdot e_{f, b, i}(t) d t \\
& +\cos \left(\vartheta_{i}\right) \int_{0}^{\Delta} v_{f, b, i}(t) \cdot n_{f, b, i}(t) d t .
\end{aligned}
$$

The correlation is maximized by

$$
\vartheta_{i}=\tan ^{-1}\left(\frac{\int_{0}^{\Delta} v_{f, b, i}(t) \cdot e_{f, b, i}(t) d t}{\int_{0}^{\Delta} v_{f, b, i}(t) \cdot n_{f, b, i}(t) d t}\right) .
$$

There are two possible $\vartheta_{i} \in\left[0^{\circ}, 360^{\circ}\right.$ [ satisfying equation (4), with $\vartheta_{i, 2}=\vartheta_{i, 1}+180^{\circ}$, one of them leading to a negative, the other to a positive correlation. The $\vartheta_{i}$ leading to a positive correlation is chosen. The method does not distinguish between prograde and retrograde particle motion as prograde Rayleigh waves are treated as retrograde waves arriving from the opposite azimuth.

[7] Although the chosen angle $\vartheta_{i}$ maximizes the correlation between $v_{f, b, i}(t)$ and $h_{f, b, i}(t)$, the correlation can be low. Thus, we make use of the correlation factor defined as

$$
c_{f, b, i}=\frac{\int_{0}^{\Delta} v_{f, b, i}(t) \cdot h_{f, b, i}(t) d t}{\sqrt{\int_{0}^{\Delta} v_{f, b, i}^{2}(t) d t \cdot \int_{0}^{\Delta} h_{f, b, i}^{2}(t) d t}} .
$$

In the case of perfect correlation between $v_{f, b, i}(t)$ and $h_{f, b, i}(t)$ this factor equals 1 . On the other side, it will vanish when the vertical and horizontal signals are not correlated at all.
[8] The signals $v_{f, b, i}(t)$ and $h_{f, b, i}(t)$ are weighted by $c_{f, b, i}^{2}$ and summed for all times $\tau_{i}$ to obtain two buffered signal windows of length $\Delta$ :

$$
\begin{aligned}
& v_{f, S}(t)=\sum_{i} c_{f, b, i}^{2} \cdot v_{f, b, i}(t) \\
& h_{f, S}(t)=\sum_{i} c_{f, b, i}^{2} \cdot h_{f, b, i}(t) .
\end{aligned}
$$

[9] Finally, the ellipticity $\epsilon$ is calculated as the square root of the ratio of the energies in the buffered signal windows $h_{f, S}(t)$ and $v_{f, S}(t)$ :

$$
\epsilon=\sqrt{\frac{\int_{0}^{\Delta}\left(h_{f, S}(t)\right)^{2} d t}{\int_{0}^{\Delta}\left(v_{f, S}(t)\right)^{2} d t}}
$$

By repeated filtering of the signal over the entire frequency range and applying the presented method the ellipticity of Rayleigh waves as a function of frequency is determined. The free parameters of the method are the width of the frequency filter $d f$ and the length of the buffered signal $\Delta$.

[10] It could be supposed that Love and body waves influence the results. In an assumed isotropic medium, Love waves are not present on the vertical component and their horizontal movements are thus uncorrelated with the vertical signal. Although incidental correlations of Love and Rayleigh waves arriving at the same time cannot be excluded, such effects will rapidly lose importance because of stacking and can thus be neglected. Arriving P- and $\mathrm{S}$-waves can be present on the vertical and horizontal components, but as their movements are not phase delayed, they will also disappear due to averaging over a sufficiently large number of windows.

\section{Application to Synthetic Noise}

[11] In order to test the method, we used a set of synthetic noise simulated for the models N101, N102, N103 and N104 of the ESG 2006 benchmark [Cornou et al., 2009]. The compressional (P) and shear wave (S) velocity profiles of these models are displayed in Figure 1.

[12] The noise synthetics were obtained in the following way: Noise sources were approximated by forces of random orientation and amplitude located $0.5 \mathrm{~m}$ below the surface [Moczo and Kristek, 2002]. The forces are randomly distributed in time and space. The time function of each source is a delta-like signal with a flat Fourier frequency-amplitude spectrum between 0.1 and $20 \mathrm{~Hz}$. In this frequency range, the associated wave field has been computed by using the wavenumber-based technique for horizontally layered structures proposed by Hisada [1994, 1995]. All types of surface and body waves are included in the simulations.

\subsection{Influence of the Parameters of the Method}

[13] As mentioned above, there are two free parameters that can be adjusted to improve the performance of the method: $d f$, the width of the frequency filter, and $\Delta$, the length of the buffered signal. To investigate the optimal parameters, we used 30 minutes of noise simulated for the model N102. The noise sources are located in the far field as well as in the local field. 

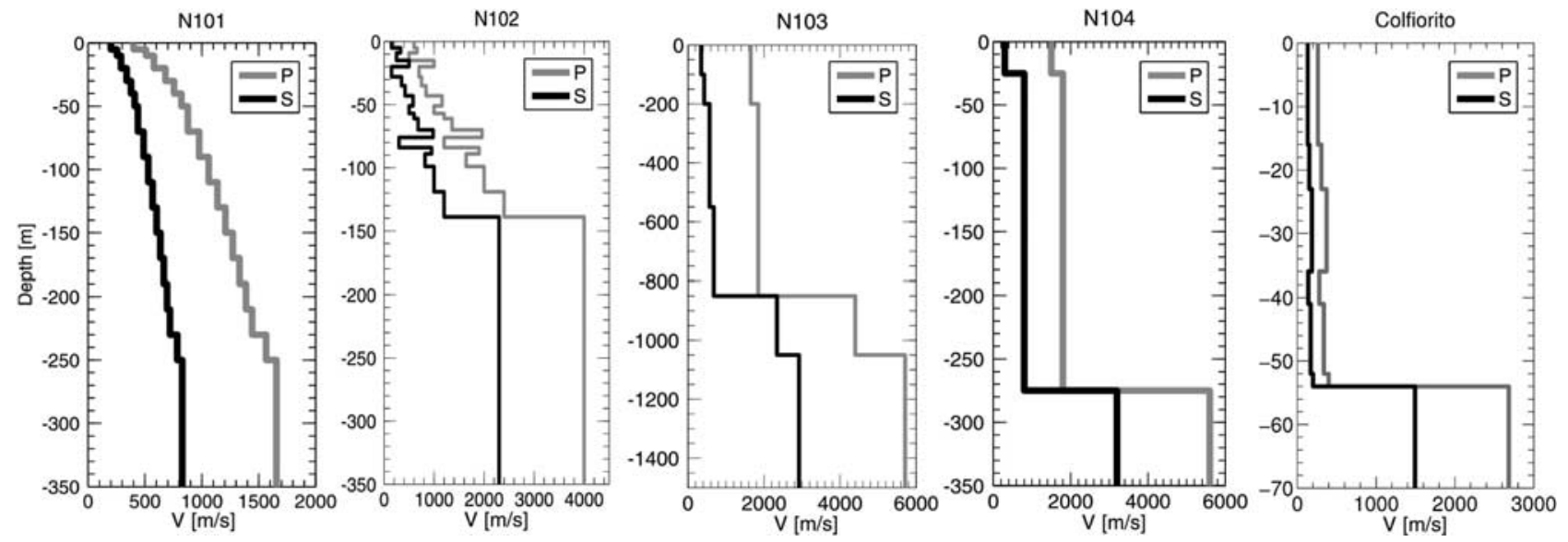

Figure 1. S- and P-wave velocity profiles for the models N101, N102, N103 and N104 [Cornou et al., 2009] and the Colfiorito borehole [Di Giulio et al., 2006].

[14] Figure 2a shows the calculated ellipticity for different bandwidths $d f$ of the frequency filter compared to the theoretical ellipticity of the model. The used bandwidths are proportional to the central frequencies. Too weak filtering $(d f=0.5 f)$ as well as too strong filtering $(d f=0.1 f)$ do not give satisfying results. An intermediate setting of $0.2 f$ reproduces the real curve the best. Therefore, we fixed the bandwidth to $0.2 f$ in the following.

[15] Figure $2 \mathrm{~b}$ shows the resulting ellipticity for different lengths $\Delta$ of the buffered signal windows in comparison to the theoretical curve. We fix $\Delta$ as a function of frequency, so that the signals consist of the same number of periods at all frequencies. As can be seen in Figure 2b, at least ten cycles are necessary for a good coverage of the signal whereas further increasing of the window length does not improve the results. Therefore, $\Delta=10 / f$ is used in the following.

\subsection{Minimum Required Signal Length and Temporal Stability of the Results}

[16] The required signal length is illustrated in Figure 2c. The same signals as in subsection 3.1 were used and the first $1800,600,300$ and 120 seconds of signal were analyzed. The results of the analysis for signals of 1800, 600 and 300 seconds only slightly differ. However, the curve obtained for 120 seconds of signal is less smooth than the other curves. At low frequencies, the signal statistics deteriorate due to the smaller number of buffered windows. It can be deduced that for sites with resonance frequencies around $1 \mathrm{~Hz}$ - a signal length of five minutes can be sufficient to measure an accurate ellipticity curve, as long as enough Rayleigh waves are present in the signal. For smaller underground resonance frequencies, the measurement has to be longer. Indeed, longer measurements improve the statistical features and the reliability of the results at all frequencies.

[17] The temporal stability of the obtained ellipticity curves is shown in Figure 2d. Five different synthetic signals of ten minutes each were simulated and the ellipticity curves were calculated for each window. The overall behavior of the curves is identical. Only the fourth tenminute-window shows some instabilities between 0.1 and $0.2 \mathrm{~Hz}$ emphasizing the need for longer signal lengths at low frequencies. For the other frequencies, the five ellipticity curves are very close. This illustrates the temporal stability of the results.

\subsection{Results for the Models N101, N103 and N104}

[18] RayDec has been applied to synthetic noise simulated for different models. The results of these simulations are shown in Figure 3. For model N101 (Figure 3a), the curve is close to the real curve over the whole frequency range, although the peak ellipticity is overestimated. For this model layout, reflected body waves can deteriorate the results. Therefore, more signal would be needed to obtain a more robust ellipticity curve. For model N103 (Figure 3b),
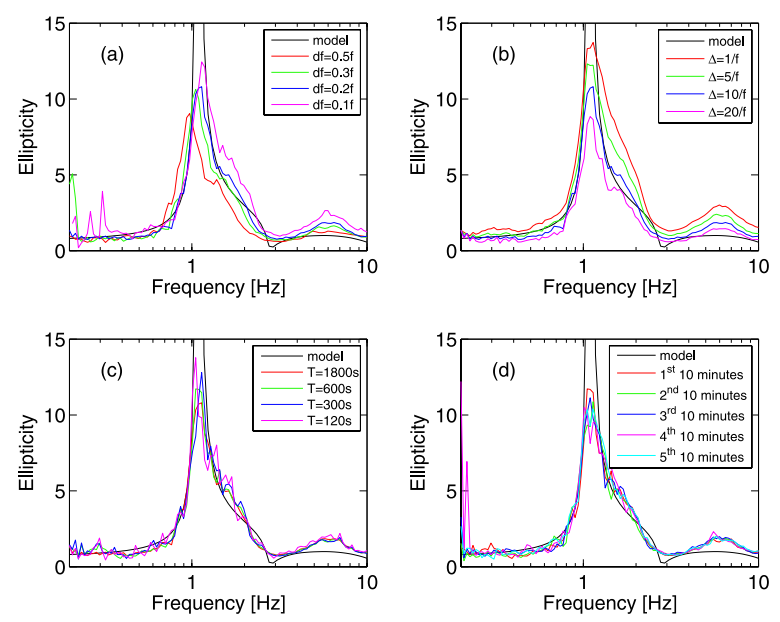

Figure 2. Sensitivity of the results with respect to the parameters of the method demonstrated on simulated seismic noise for the N102 model. The real ellipticity curve of the model is included for comparison. Curves of Figures 2a and $2 \mathrm{~b}$ were calculated for 30 minutes of simulated seismic noise. (a) Effect of the filter bandwidth $d f$ on the resulting ellipticity curve $(\Delta=10 / f)$ for $d f=0.5 f, d f=0.3 f, d f=0.2 f$ and $d f=0.1 f$. (b) Effect of the length $\Delta$ of the buffered signal $(d f=0.2 f)$ for $\Delta=1 / f, \Delta=5 / f, \Delta=10 / f$ and $\Delta=20 / f$. (c) Effect of the length of the signals (time length $T$, $d f=0.2 f, \Delta=10 / f$ ) for $T=1800 s, T=600 s, T=300 s$ and $T=120 \mathrm{~s}$. (d) Analysis of five different signals of ten minutes each $(d f=0.2 f, \Delta=10 / f)$. 

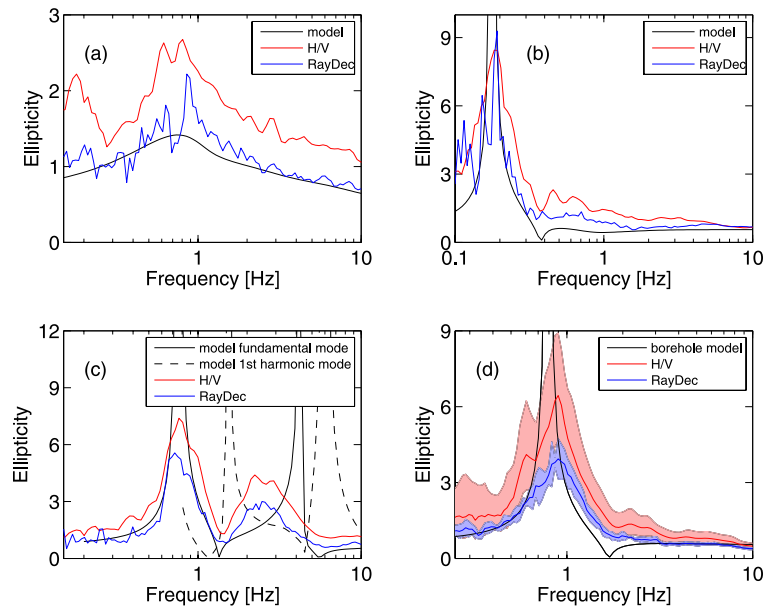

Figure 3. Ellipticity curves obtained for ten minutes of synthetic noise simulated for three different underground models ((a) N101, (b) N103 and (c) N104) and for one hour of real noise from Colfiorito basin (d). The used parameters are $d f=0.2 f$ and $\Delta=10 / f$. In the four graphs, the results are compared to the real ellipticity curves of the fundamental mode and the first harmonic mode (only in Figure 3c) and to the results obtained by the $\mathrm{H} / \mathrm{V}$ technique. (d) Curve also contains the error ranges of the H/V and RayDec curves.

there is a good agreement between the simulated and the theoretical curves around the resonance frequency and at higher frequencies, with some minor deviations between 0.3 and $2 \mathrm{~Hz}$. Below $0.15 \mathrm{~Hz}$, the curve differs considerably from the theoretical curve. Model N104 (Figure 3c) exhibits some unfavorable behavior, as the fundamental mode and the first harmonic mode of Rayleigh wave motion overlap above 1.4 Hz. RayDec cannot distinguish between two modes. Therefore, its results are a mixture of the two modes in this frequency range. For lower frequencies, RayDec reproduces well the theoretical curve around the first peak of the fundamental mode. The $\mathrm{H} / \mathrm{V}$ curve follows the same trend, but the absolute values of the RayDec curve better reproduce the right flank of the fundamental peak.

[19] It can be stated that, in general, the results of RayDec are much closer to the theoretical curves than the results obtained with the H/V method. However, RayDec regularly fails in reproducing very small ellipticity values. At these points, the horizontal components of the Rayleigh wave vanish. Therefore, it is problematic to find horizontal signals correlated to the vertical signal. The same reasoning is valid for the peak frequency ellipticity in the case of a theoretically vanishing vertical component. This explains why the peak ellipticity cannot be satisfactorily estimated. On the other hand, small ellipticities can also be hidden by the presence of higher Rayleigh wave modes. In cases where higher Rayleigh wave modes are close to the fundamental modes, RayDec only indicates an apparent ellipticity which cannot be simply related with the true ellipticity of each mode.

\section{Application to Real Noise Data}

[20] To test the RayDec method on real data, we applied it to one hour of seismic noise recorded in the center of
Colfiorito basin in central Italy. Colfiorito basin is a wellknown site which has been extensively investigated by different geophysical methods [Di Giulio et al., 2006]. The theoretical ellipticity curve is calculated on the basis of S- and P-wave velocity profiles obtained by a borehole measurement (see Figure 1). The parameters used were $d f=0.2 f$ and $\Delta=10 / f$. The hour of signal was cut into six ten-minute signals which were analyzed separately. The resulting curves were then averaged. When comparing with the theoretical ellipticity curve, one should keep in mind that a borehole measurement is made at a selected point, whereas a seismic noise record is influenced by the whole surrounding underground structure. The RayDec curve is in better agreement with the theoretical curve than the $\mathrm{H} / \mathrm{V}$ curve, especially on the slopes of the fundamental resonance frequency peak (Figure 3d). However, both the H/V and RayDec curve's peak frequencies are shifted with respect to the borehole model's peak frequency.

\section{Conclusion}

[21] RayDec is a fast and simple method to determine the ellipticity of Rayleigh waves. As it suppresses Love and body waves efficiently, its results are closer to theory than $\mathrm{H} / \mathrm{V}$ results. The necessary signal length depends on the resonance frequency of the underground structure, but some minutes of signal can be sufficient to get satisfying results, as long as enough Rayleigh waves are present in the wave field, although longer signal lengths are preferable. However, as it is a one-sensor technique, RayDec gives two possible azimuths and cannot distinguish between prograde and retrograde particle motion. By coupling RayDec with array measurements the correct azimuth and the sense of particle motion could be retrieved. Besides that, it could be envisaged to estimate the proportion of Rayleigh waves in the wave field by comparing RayDec results including only Rayleigh waves with $\mathrm{H} / \mathrm{V}$ measurements including all wave types. The future work consists of trying to improve the inversion of S-wave velocity profiles by using RayDec's ellipticity estimations. This is a non-trivial endeavor which will be addressed in a future paper.

[22] Acknowledgments. Noise synthetics were performed at the Service Commun de Calcul Intensif (SCCI) of Grenoble observatory, France (OSUG). Ambient noise data from Colfiorito site were collected during the SESAME project (EVG1-CT-2000-00026). This work is supported by the NERIES European project and the ASIRAN ANR project. We would like to thank two anonymous reviewers whose comments helped to improve the manuscript.

\section{References}

Arai, H., and K. Tokimatsu (2004), S-wave velocity profiling by inversion of microtremor H/V spectrum, Bull. Seismol. Soc. Am., 94, 53-63.

Arai, H., and K. Tokimatsu (2005), S-wave velocity profiling by joint inversion of microtremor dispersion curve and horizontal-to-vertical (H/V) spectrum, Bull. Seismol. Soc. Am., 95, 1766-1778, doi:10.1785/ 0120040243 .

Asmussen, J. C. (1997), Modal analysis based on the random decrement technique, Ph.D. thesis, Univ. of Aalborg, Aalborg, Denmark.

Bonnefoy-Claudet, S., A. Köhler, C. Cornou, M. Wathelet, and P.-Y. Bard (2008), Effects of Love waves on microtremor H/V ratio, Bull. Seismol. Soc. Am., 98, 288-300, doi:10.1785/0120070063.

Boore, D. M., and M. N. Toksöz (1969), Rayleigh wave particle motion and crustal structure, Bull. Seismol. Soc. Am., 59, 331-346.

Cornou, C., M. Ohrnberger, D. M. Boore, K. Kudo, and P.-Y. Bard (2009), Derivation of structural models from ambient vibration array recordings: Results from an international blind test, in ESG 2006: 
Third International Symposium on the Effects of Surface Geology on Seismic Motion, vol. 2, edited by P.-Y. Bard et al., Lab. Cent. des Ponts et Chaussées, Paris, in press.

Di Giulio, G., C. Cornou, M. Ohrnberger, M. Wathelet, and A. Rovelli (2006), Deriving wavefield characteristics and shear-velocity profiles from two-dimensional small-aperture arrays analysis of ambient vibrations in a small-size alluvial basin, Colfiorito, Italy, Bull. Seismol. Soc. Am., 96, 1915-1933, doi:10.1785/0120060119.

Dunand, F. (2005), Pertinence du bruit de fond sismique pour la caractérisation dynamique et l'aide au diagnostic sismique des structures de génie civil, Ph.D. thesis, Univ. Joseph Fourier, Grenoble, France.

Fäh, D., F. Kind, and D. Giardini (2001), A theoretical investigation of average $\mathrm{H} / \mathrm{V}$ ratios, Geophys. J. Int., 145, 535-549.

Fäh, D., F. Kind, and D. Giardini (2003), Inversion of local S-wave velocity structures from average $\mathrm{H} / \mathrm{V}$ ratios, and their use for the estimation of site-effects, J. Seismol., 7, 449-467.

Hisada, Y. (1994), An efficient method for computing Green's functions for a layered halfspace with sources and receivers at close depths, Bull. Seismol. Soc. Am., 84, 1456-1472.

Hisada, Y. (1995), An efficient method for computing Green's functions for a layered halfspace with sources and receivers at close depths (part 2), Bull. Seismol. Soc. Am., 85, 1080-1093.

Köhler, A., M. Ohrnberger, and F. Scherbaum (2006), The relative fraction of Rayleigh and Love waves in ambient vibration wavefields at different European sites, ESG 2006: Third International Symposium on the Effects of Surface Geology on Seismic Motion, vol. 1, edited by P.-Y. Bard et al., pp. 351-360, Lab. Cent. des Ponts et Chaussées, Paris.

Malischewsky, P. G., and F. Scherbaum (2004), Love's formula and H/Vratio (ellipticity) of Rayleigh waves, Wave Motion, 40, 57-67.

Michel, C., P. Guéguen, and P.-Y. Bard (2008), Dynamic parameters of structures extracted from ambient vibration measurements: An aid for the seismic vulnerability assessment of existing buildings in moderate seismic hazard regions, Soil Dyn. Earthquake Eng., 28, 593-604.

Moczo, P., and J. Kristek (2002), FD code to generate noise synthetics, Sesame Rep. D02.09, IGSAS, Bratislava. (Available at http://SESAMEFP5.obs.ujf-grenoble.fr)

Nakamura, Y. (1989), A method for dynamic characteristics estimation of subsurface using microtremor on the ground surface, Q. Rep. RTRI, 30, $25-33$.

Picozzi, M., S. Parolai, and S. M. Richwalski (2005), Joint inversion of H/V ratios and dispersion curves from seismic noise: Estimating the S-wave velocity of bedrock, Geophys. Res. Lett., 32, L11308, doi:10.1029/ 2005GL022878.

Scherbaum, F., F. K. Hinzen, and M. Ohrnberger (2003), Determination of shallow shear wave velocity profiles in the Cologne, Germany area using ambient vibrations, Geophys. J. Int., 152, 597-612.

P.-Y. Bard, C. Cornou, and M. Hobiger, LGIT, BP 53, F-38041 Grenoble CEDEX 9, France. (manuel.hobiger@obs.ujf-grenoble.fr)

N. Le Bihan, GIPSA-Lab, BP 46, F-38402 Grenoble CEDEX, France. 